\title{
Lack of Family Support and Psychopathy Facilitates Antisocial Punishment Behavior in College Students
}

\author{
Keita Masui $^{1 *}$, Shouichi Iriguchi ${ }^{2}$, Miki Terada $^{1}$, Michio Nomura $^{3}$, Mitsuhiro Ura ${ }^{1}$ \\ ${ }^{1}$ Department of Human Sciences, Graduate School of Integrated Arts and Science, \\ Hiroshima University, Hiroshima, Japan \\ ${ }^{2}$ Faculty of Integrated Arts and Science, Hiroshima University, Hiroshima, Japan \\ ${ }^{3}$ Graduate School of Education, Kyoto University, Kyoto, Japan \\ Email: "keita-masui@hiroshima-u.ac.jp
}

Received December 28 ${ }^{\text {th }}, 2011$; revised January $19^{\text {th }}, 2012$; accepted February $23^{\text {rd }}, 2012$

\begin{abstract}
Both a lack of social support and psychopathy show a positive association with aggressive behavior. This study investigated whether a perceived lack of family support and psychopathy would facilitate "antisocial punishment behavior," which was defined as punishment behavior to cooperators in a trust game. The participants were four groups of university students with low or high levels of psychopathy who had also reported low or high levels of family support $(N=48)$. In a trust game played on a computer, participants were given the chance to reduce the compensation as a punishment of their (simulated) partners based on whether they were cooperators or non-cooperators. We found that high-psychopathy participants with low family support gave cooperators significantly more punishment than did participants with low psychopathy and high family support. The study indicates that an interaction between a lack of family support and psychopathy contributes to aggressive behavior, such as antisocial punishment behavior.
\end{abstract}

Keywords: Family Support; Psychopathy; Aggression; Antisocial Punishment; Decision-Making

\section{Introduction}

Social support is necessary because people are social animals and live in mutual interaction with other people. Effective social support is able not only to buffer psychological distress after terrible natural disasters (Lowe, Chan, \& Rhodes, 2010), but also to protect people from mental disorder, disease, and even death (e.g., Broadhead et al., 1983). Conversely, lack of social support has been associated with increased risk for morbidity, mortality, and psychological distress (e.g., Hawkley, Masi, Berry, \& Cacioppo, 2006; House, Landis, \& Umberson, 1988). Moreover, perceptions of low parental support predicted childhood aggression, particularly for boys (Garbarino, 1999; Patterson, Kupersmidt, \& Griesler, 1990).

One reason why a lack of parental support facilitates aggression may be that it is assumed to be social rejection. Previous research has indicated that interpersonal rejection affected not only aggressive behavior but also mediators of aggression, such as anger and the derogation of other people (for a review, see Leary, Twenge, \& Quinlivan, 2006). Because support from family or close friends is generally anticipated and socially normative (Inaba, 1998), the lack of such support could be taken as social rejection for the lateral receiver of the support.

A lack of parental support facilitates aggressive behavior. This effect is possibly accentuated for people with high dispositional aggressiveness. Among dispositions relevant for aggressiveness, psychopathy is one of the most studied (e.g., Coyne \& Thomas, 2008). Psychopathy is defined as a constellation of affective, interpersonal, and behavioral characteristics, including a lack of empathy, guilt, or remorse, egocentricity, irresponsibility, shallow emotions, and impulsivity (Hare, 1998).

\footnotetext{
*Corresponding author.
}

There are two related but distinct components of psychopathy: primary and secondary. Primary psychopathy is predominantly characterized by interpersonal and affective problems, such as cruelty and a lack of fear or empathy, while secondary psychopathy is characterized by features such as impulsivity and neuroticism (e.g., Hare, Harpur, Hakstian, Forth, \& Hart, 1990; Levenson, Kiehl, \& Fitzpatrick, 1995; Lykken, 1957). In a previous study, primary and secondary psychopathy in non-clinical adolescents showed significant positive associations with both direct and indirect aggression (Coyne \& Thomas, 2008). Furthermore, hostile/reactive aggression, which has been described as an emotion-driven, impulsive, defensive response to a perceived threat (Berkowitz, 1989) was related to each component of psychopathy (Reidy, Zeichner, Miller, \& Martinez, 2007).

Thus, an interaction between a lack of social support and psychopathy might be expected to facilitate aggressive behavior. However, a link has not been clarified between a lack of social support, psychopathy, and aggressive behavior in a non-clinical population. In this study, we use family support as the principal social support because it has the highest expected influence in the contextual model of social support (Inaba, 1998), and low levels of parental support predict childhood aggression (Garbarino, 1999; Patterson et al., 1990).

In this study, the measures of participants' aggressive behavior were their responses in series of trust games played with a (simulated) partner over a computer connection (de Quervain et al., 2004; Masui, Iriguchi, Nomura, \& Ura, 2011). In the trust games, participants were given the chance to "punish" by reducing the payout for partners who were cooperators or noncooperators. In a previous study, punishing a cooperator was said to represent "antisocial punishment behavior" (Rand, Armao, Nakamaru, \& Ohtsuki, 2010). In the present study, we used antisocial punishment behavior as an index of the partici- 
pants' aggressive behavior.

The purpose of this study is to investigate the association between a perceived lack of family support, psychopathy, and aggressive behavior in a non-clinical population. Earlier findings led to the following hypothesis: participants with high psychopathy who also have less family support should give cooperators more punishment, as compared to participants with high family support or low psychopathy.

\section{Method}

\section{Participants}

Participants ( $N=48 ; 20$ men and 28 women) were recruited for the present experiment from a total initial sample of 111 Japanese university students (52 men and 59 women) who completed the Quality of Relationship Inventory (QRI; Pierce, Sarason, \& Sarason, 1991) and the Levenson Self-Report Psychopathy scale (LSRP; Levenson et al., 1995); their mean age was 18.85 years $(\mathrm{SD}=0.55)$. Participants gave written informed consent prior to taking the questionnaires and participating in the experiment. The Ethics and Safety Committee of Hiroshima University approved the study protocol.

The participants were divided into four groups according to the medians of the distribution of scores on the QRI (median = 28 ) and the LSRP (median = 48): low support (low score on QRI) \& low psychopathy (low score on LSRP); low support \& high psychopathy; high support \& low psychopathy; high support \& high psychopathy.

\section{Measures}

Family support. Family support was assessed by the QRI. The QRI is a questionnaire which assesses the perceived availability of social support from specific relationships. It has three subscales: social support, conflict, and depth of specific relationships. We revised the social support factor in a Japanese version of the QRI developed by Ura and Takano (1995). The QRI in this study consisted of 7 items rated on a 5-point scale (from disagree to agree), which had adequate reliability $(\alpha=0.72)$.

Psychopathy. The LSRP scale is a 26-item questionnaire designed to measure psychopathic traits in noon-clinical populations. Each item is a statement rated on a 4-point scale (from disagree strongly to agree strongly). The LSRP scale has two subscales: primary and secondary psychopathy. The primary psychopathy subscale consists of 16 items pertaining to manipulation, egocentricity, lack of empathy, and remorse. The secondary psychopathy subscale consists of 10 items relating to impulsivity, quick-temperedness, and poor behavioral control. A Japanese version of the LSRP scale was developed through back translation of the items (Sugiura \& Sato, 2005). It demonstrated the same factor structure as the original, with adequate test-retest reliability and construct validity (Osumi, Kanayama, Sugiura, \& Ohira, 2007). The coefficient alphas for this study were 0.78 for the total LSRP scale, 0.80 for the primary psychopathy scale, and 0.57 for the secondary psychopathy scale; these values are approximately equivalent to those provided by Masui and Nomura (2011).

The QRI scores were significantly different between the high support and the low support groups, and the scores of the total LSRP, primary psychopathy, and secondary psychopathy were also significantly different between the high psychopathy and the low psychopathy groups (see Table 1).
Demographic variables. Sex and age of all participants were investigated as demographic variables.

\section{Procedure}

The paradigm used in the present study was a computerized trust game designed to assess punishment behavior during both "fair" and "unfair" rounds. Similar to previous studies (de Quervain et al., 2004; Masui et al., 2011), participants were told that this study was examining economic decision-making. They would be playing a point distribution game on the computer against four different human players (in reality, all participants were playing against the computer). One participant per session arrived at the laboratory and was seated in front of a laptop computer. To ensure that the participants believed they were playing against other people, we communicated with a collaborator via a wireless transceiver, as if to suggest that other participants were playing the game at the same time but in a different laboratory.

Participants were told that they would be playing with one partner per round, and that they could earn points based on their decisions during the experiment. They were also told that the points earned during the experiment would be converted into money at the end of the experiment ( 10 points $=30$ yen $)$.

The trust game itself consisted of three steps. At the beginning of the experiment, the participant and the "partner" received an endowment of 10 points. During the first step, the participant decided whether to transfer the allotment of 10 points to the partner or transfer nothing at all. If the participant transferred the 10 points, the points were quadrupled, meaning that the partner received 40 points in addition to the original 10-point endowment. If the participant transferred nothing, both players remained at 10 points. During the second step, the partner decided whether to transfer half of their points (either 5 or $25)$ to the participant. If the partner transferred half of their points, then the participant received exactly the same number of points transferred. After that, both players received an additional endowment of 20 points. During the third step, the participant got the option of assigning penalty points to the partner, such that the partner lost double the points assigned as a penalty. The participants punished their partners in either a costly or a non-costly situation. In the costly situation, for example, if the participant assigned 5 points as a penalty, then 5 points were deducted from the participant and 10 points were deducted from the partner. In the non-costly situation, for example, if the participant assigned 5 points as a penalty, then no points were deducted from the participant and 10 points were deducted from the partner. In all situations, participants could assign their partner between 0 and 20 penalty points, and they wrote down the penalty points they assigned on a paper. During two of the four rounds of the game, participants were treated in a fair manner (receiving half of the points back), whereas for the other two rounds, they were treated unfairly (receiving no points back). During two of the four rounds, participants performed in the costly situation, and for the other two rounds they performed in the non-costly situation. The partner's choice (fair/unfair) and situation (costly/non-costly) were randomly determined on a per-trial basis, and different random orders were administered to each participant. None of the participants had prior knowledge of the rules at any step.

Following each round, following participants completed a questionnaire that measured how they felt in terms of fairness, 
Table 1.

Demographic data, QRI scores, and LSRP scores.

\begin{tabular}{|c|c|c|c|c|c|c|c|c|c|c|}
\hline & \multicolumn{4}{|c|}{ Low support } & \multicolumn{4}{|c|}{ High support } & \multirow{3}{*}{$\mathrm{F}(3,44)$} & \multirow{3}{*}{$P$} \\
\hline & \multicolumn{2}{|c|}{$\begin{array}{l}\text { Low psychopathy } \\
\qquad(\mathrm{n}=7)\end{array}$} & \multicolumn{2}{|c|}{$\begin{array}{l}\text { High psychopathy } \\
\quad(\mathrm{n}=18)\end{array}$} & \multicolumn{2}{|c|}{$\begin{array}{l}\text { Low psychopathy } \\
\quad(\mathrm{n}=16)\end{array}$} & \multicolumn{2}{|c|}{$\begin{array}{l}\text { High psychopathy } \\
\quad(\mathrm{n}=7)\end{array}$} & & \\
\hline & Mean & S.D. & Mean & S.D. & Mean & S.D. & Mean & S.D. & & \\
\hline Age & 18.86 & 0.69 & 18.83 & 0.62 & 18.88 & 0.50 & 18.86 & 0.38 & 0.02 & N.S. \\
\hline QRI & 24.29 & 2.63 & 31.89 & 2.25 & 25.06 & 1.73 & 31.86 & 2.80 & 40.12 & $<.001^{\mathrm{a}}$ \\
\hline LSRP total score & 42.71 & 3.15 & 41.67 & 3.82 & 54.69 & 5.40 & 56.71 & 9.03 & 25.65 & $<.001^{\mathrm{b}}$ \\
\hline LSRP primary psychopathy score & 26.86 & 2.91 & 24.83 & 3.09 & 33.19 & 4.48 & 34.57 & 10.15 & 10.85 & $<.001^{\mathrm{b}}$ \\
\hline LSRP secondy psychopathy score & 15.86 & 1.86 & 16.83 & 3.02 & 21.50 & 3.88 & 22.14 & 3.98 & 9.54 & $<.001^{\mathrm{b}}$ \\
\hline
\end{tabular}

Note: QRI = Quality of Relationship Inventory; LSRP = Levenson Self-Report Psychopathy scale; ${ }^{\mathrm{a} l o w}$ support $<$ high support; ${ }^{\mathrm{b}}$ low psychopathy $<$ high psychopathy.

gratification, kindness, hostility, anger, and irritation with regards to the partner's choice, on an analogue rating scale ranging from 1 (not at all) to 7 (strongly). This questionnaire was a modified version of an emotional questionnaire used in previous studies (de Quervain et al., 2004; Masui et al., 2011). Participants subsequently completed another questionnaire that measured how they felt regret, pity, pleasure, or feelings of gloating after they had punished a partner, each rated on a seven-point scale. This questionnaire was a modified version of a questionnaire measuring empathy or taking pleasure in other's unhappiness used in a previous study (Sawada, 2008).

After all four rounds, participants were debriefed and were paid 500 Yen (approximately 7 US\$) for their participation in the study.

\section{Data Analysis}

We carried out a correlation analysis and repeated and mixed model analyses of variance (ANOVAs) with Support (high/low) and Psychopathy (high/low) as the between-subjects factor, and partner Fairness (fair/unfair) and Situation (costly/non-costly) as within-subject factors. The amount of punishment administered was the dependent variable. Bonferroni test was used for post-hoc comparisons.

\section{Results}

\section{Manipulation Check}

Compared to the unfair partner, participants rated the fair partner as more likely to make them feel like the situation was fair (Ms 6.00 vs. 2.84), feel satisfaction with the situation (Ms 6.55 vs. 2.48 ), and feel kindness toward the partner ( $M$ s 5.94 vs. 2.65, $p \mathrm{~s}<0.001, \eta_{p}^{2}>0.72$ ). Participants also rated the unfair partner as more likely to make them feel hostile (Ms 1.19 vs. 3.56 ), angry ( $M$ s 1.19 vs. 3.85 ), and irritated (Ms 1.18 vs. 3.72, ps $\left.<0.001, \eta_{p}^{2}>0.57\right)$. There were no significant main effects of Support, or Psychopathy, or Situation ( $p s>0.11, \eta_{p}^{2}<$ $0.05)$.

\section{Correlation of Family Support, Psychopathy, and the Amount of Punishment}

Table 2 shows the Pearson's correlation coefficients for family support, psychopathy, and the amount of punishment. There was a marginal negative correlation between family support and primary psychopathy $(r=-0.28, p=0.06)$. Furthermore, primary psychopathy was positively associated with the number of penalty points allocated to unfair partnerss in the costly situation $(r=0.35, p<0.05)$.

\section{Analysis of Antisocial Punishment Behavior}

Figure 1 shows the mean amount of punishment administered for each participant group. Repeated measures ANOVAs revealed significant main effects of partner Fairness and Situation, $F_{\mathrm{s}}(1,44)>25.03, p \mathrm{~s}<0.001, \eta_{p}^{2}>0.36$, and a significant interaction effect between partner Fairness and Situation $F(1,44)=13.30, p<0.01, \eta_{p}^{2}=0.23$. Participants punished the unfair partner rather than the fair partner both in costly and non-costly situations $(p<.001)$.

There was also a significant interaction between Support, Psychopathy, and partner Fairness, $F(1,44)=5.65, p<0.05$, $\eta_{p}^{2}=0.11$. A post-hoc test showed that participants in the low support \& high psychopathy group punished the fair partner significantly more than participants in the high support \& high psychopathy, and low support \& low psychopathy groups (low support \& high psychopathy, $M=4.00$; high support $\&$ high psychopathy; $M=0.64$, low support \& low psychopathy, $M=$ $0.36, p<0.05)$. On the other hand, there was no main effect of Support or Psychopathy, and no significant interaction between Support and partner Fairness, Psychopathy and partner Fairness, and Support, Psychopathy, partner Fairness, and Situation, Fs $(1,44)<1.92$, ps $>0.17$.

\section{Discussion}

The goal of the present study was to examine whether a lack of family support and psychopathy would facilitate antisocial punishment behavior in college students. We recruited participants who had low and high levels of perceived family support with low and high psychopathy, and conducted an experiment using a series of trust games. The results of correlation analyses showed that a lack of family support was marginally related to primary psychopathy. Moreover, repeated measures ANOVAs showed a significant interaction between family Support, Psychopathy, and partner Fairness. A post-hoc test revealed that high-psychopathy participants with low family support exhibited significantly greater levels of antisocial punishment behavior, as compared to low-psychopathy participants with high family support.

Some previous research has indicated a relationship between a lack of social support and aggressive behavior (e.g., Kirkpatrick, Waugh, Valencia, \& Webster, 2002; Twenge, Baumeister, Tice, \& Stucke, 2001; Warburton, Williams, \& Cairns, 
Table 2.

Correlation of family support, psychopathy, and amount of punishment.

\begin{tabular}{lccccccc}
\hline & 1 & 2 & 3 & 4 & 5 & 6 & 7 \\
\hline 1. QRI & - & & & & & & \\
2. Primary psychopathy & $-.28^{\dagger}$ & - & & & & & \\
3. Secondary psychopathy & -.10 & .24 & - & & & & \\
4. Penalty points (FC) & -.14 & .04 & -.13 & - & & & \\
5. Penalty points (FN) & -.22 & .24 & .07 & $.34^{*}$ & - & & \\
6. Penalty points (UC) & -.12 & $.35^{*}$ & .17 & .20 & .04 & - & \\
7. Penalty points (UN) & -.18 & .23 & .07 & .07 & .08 & .06 & - \\
\hline
\end{tabular}

Notes: $\mathrm{F}=$ fair partner; $\mathrm{U}=$ unfair partner; $\mathrm{C}=$ costly situation; $\mathrm{N}=$ no-costly situation. Significance levels of correlations are denoted by ${ }^{*} p<0.05$ and ${ }^{\dagger} p=0.06$.

(a)

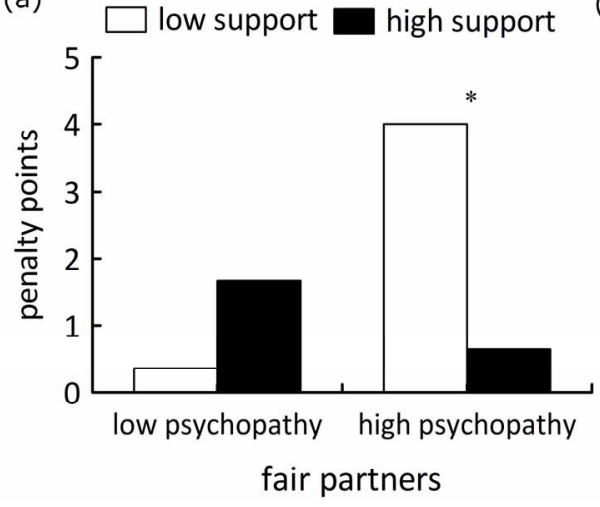

(b)

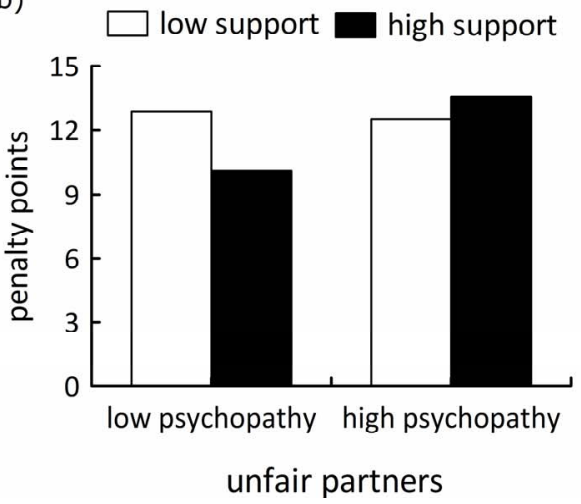

Figure 1.

(a) Penalty points allocated to fair partners; (b) Penalty points allocated to unfair partners. Low-psychopathy participants are represented on the left side and high-psychopathy participants on the right. The white bar indicates the low-family-support participants, and the black bar indicates the high-family-support participants. The statistical result for the between-groups comparison is given for each condition $\left({ }^{*} p<0.05\right)$.

2003). For example, Warburton et al. (2003) showed a link between social rejection and aggression. Participants experienced rejection or inclusion in a virtual ball-tossing game and also experienced control (or no control) over unpleasant noise. Participants then had the opportunity to aggress against an innocent target. They were told that the target person did not like spicy foods and that they could assign him or her to eat hot sauce. The results showed that rejected participants who had no control over the noise were the most aggressive, assigning the target person to eat four times as much hot sauce as participants in the other conditions. Thus, when rejected participants could not control an aversive situation, they were more aggressive toward an innocent target. Similarly, a lack of parental support predicted childhood aggression for boys (Garbarino, 1999; Patterson et al., 1990). Furthermore, there are positive correlations between psychopathy and various forms of aggression such as direct, indirect, and hostile/reactive aggression (Coyne $\&$ Thomas, 2008; Reidy et al., 2007). The findings in our present study are consistent with these previous findings. Overall, the present study offers a unique insight that an interaction between a lack of family support and psychopathy would facilitate or amplify aggressive behavior, such as antisocial punishment behavior.

The results of the present study are limited because the sam- ple size is small and limited to college students. We only investigated the relationship between a lack of family support, psychopathy, and antisocial punishment behavior. Because there is a positive correlation between family support and support from friends (Ura \& Takano, 1995), it is possible that the perceived support from other relationships would also affect antisocial punishment behavior. Furthermore, it is possible that other social support (e.g., close friends or a romantic partner) would buffer the lack of family support. We did not assess the psychological effect of lack of family support. Leary et al. (2006) proposed possible reasons why interpersonal rejection leads to anger and aggression: interpersonal rejection is a source of pain or frustration, a threat to self-esteem, and there is mood improvement following aggression. Further research that involves a larger or more diverse sample and looks at various types of social support would be required to provide additional support for our proposition about the relationship between a lack of social support, psychopathy, and antisocial punishment behavior.

In summary, the present study is the first to indicate that lack of social support, such as family support and psychopathy interact with each other to facilitate antisocial punishment behavior in a non-clinical population. The current findings provide further insights into aggressive and antisocial behavior. 


\section{REFERENCES}

Berkowitz, L. (1989). Frustration-aggression hypothesis: Examination and reformulation. Psychological Bulletin, 106, 59-73.

doi:10.1037/0033-2909.106.1.59

Broadhead, W. E., Kaplan, B. H., James, S. A., Wagner, E. H., Schoenbach, V. J., Grimson, R., et al. (1983). The epidemiological evidence for a relationship between social support and health. American Journal of Epidemiology, 117, 521-537.

Coyne, S. M., \& Thomas, T. J. (2008). Psychopathy, aggression, and cheating behavior: A test of the Cheater-Hawk hypothesis. Personality and Individual Differences, 44, 1105-1115. doi:10.1016/j.paid.2007.11.002

De Quervain, D. J. F., Fischbacher, U., Treyer, V., Schellhammer, M., Schnyder, U., Buck, A. et al. (2004). The neural basis of altruistic punishment. Science, 305, 1254-1258. doi:10.1126/science.1100735

Garbarino, J. (1999). Lost boys: Why our sons turn violent and how we can save them. San Francisco: Jossey-Bass.

Hare, R. D. (1998). Psychopaths and their nature: Implications for the mental health and criminal justice systems. In T. Millon, E. Simonsen, M. Biket-Smith, \& R. D. Davis (Eds.), Psychopathy: Antisocial, criminal and violent behavior (pp. 188-212). New York: Guilford Press.

Hare, R. D., Harpur, T. J., Hakstian, A. R., Forth, A. E., \& Hart, S. D. (1990). The revised psychopathy checklist: Reliability and factor structure. Psychological Assessment, 2, 338-341. doi:10.1037/1040-3590.2.3.338

Hawkley, L. C., Masi, C. M., Berry, J. D., \& Cacioppo, J. T. (2006). Loneliness is a unique predictor of age-related differences in systolic blood pressure. Psychology and Aging, 21, 152-164. doi:10.1037/0882-7974.21.1.152

House, J. S., Landis, K. R., \& Umberson, D. (1988). Social relationships and health. Science, 241, 540-545. doi:10.1126/science.3399889

Inaba, A. (1998). The theoretical model of social support. In Y. Matsui, \& M. Ura (Eds.), Psychological science of human support (pp. 151176). Tokyo: Seishinshobo.

Kirkpatrick, L. A., Waugh, C. E., Valencia, A., \& Webster, G. D. (2002). The functional domain specificity of self-esteem and the differential prediction of aggression. Journal of Personality and Social Psychology, 82, 756-767. doi:10.1037/0022-3514.82.5.756

Leary, M. R., Twenge, J. M., \& Quinlivan, E. (2006). Interpersonal rejection as a determinant of anger and aggression. Personality and Social Psychology Review, 10, 111-132. doi:10.1207/s15327957pspr1002_2

Levenson, M. R., Kiehl, K. A., \& Fitzpatrick, C. M. (1995). Assessing psychopathic attributes in a noninstitutionalized population. Journal of Personality and Social Psychology, 68, 151-158. doi:10.1037/0022-3514.68.1.151

Lowe, S. R., Chan, C. S., \& Rhodes, J. E. (2010). Pre-hurricane perceived social support protects against psychological distress: A longitudinal analysis of low-income mothers. Journal of Consulting and
Clinical Psychology, 78, 551-560. doi:10.1037/a0018317

Lykken, D. T. (1957). A study of anxiety in the sociopathic personality. Journal of Abnormal Psychology, 55, 6-10. doi:10.1037/h0047232

Masui, K., Iriguchi, S., Nomura, M., \& Ura, M. (2011). Amount of altruistic punishment accounts for subsequent emotional gratification in participants with primary psychopathy. Personality and Individual Differences, 51, 823-828. doi:10.1016/i.paid.2011.07.006

Masui, K., \& Nomura, M. (2011). The effects of reward and punishment on response inhibition in non-clinical psychopathy. Personality and Individual Differences, 50, 69-73.

doi:10.1016/j.paid.2010.08.024

Osumi, T., Kanayama, N., Sugiura, Y., \& Ohira, H. (2007). Validation of the Japanese primary and secondary psychopathy scales. The Japanese Journal of Personality, 16, 117-120. doi:10.2132/personality. 16.117

Patterson, C. J., Kupersmidt, J. B., \& Griesler, P. C. (1990). Children's perceptions of self and of relationships with others as a function of sociometric status. Child Development, 61, 1335-1349. doi: $10.2307 / 1130746$

Pierce, G. R., Sarason, I. G., \& Sarason, B. R. (1991). General and relationship-based perceptions of social support: Are two constructs better than one? Journal of Personality and Social Psychology, 61, 1028-1039. doi:10.1037/0022-3514.61.6.1028

Rand, D. G., Armao IV, J., Nakamura, M., \& Ohtsuki, H. (2010). Antisocial punishment can prevent the co-evolution of punishment and cooperation. Journal of Theoretical Biology, 265, 624-632. doi:10.1016/j.jtbi.2010.06.010

Reidy, D. E., Zeichner, A., Miller, J. D., \& Martinez, M. A. (2007). Psychopathy and aggression: Examining the role of psychopathy factors in predicting laboratory aggression under hostile and instrumental conditions. Journal of Research in Personality, 41, 12441251. doi:10.1016/j.jrp.2007.03.001

Sawada, M. (2008). The role of envy and personality traits on Schadenfreude: Links with guilt, self-esteem, and narcissism. The Japanese Journal of Research on Emotion, 16, 36-48. doi: $10.4092 /$ jsre. 16.36

Sugiura, Y., \& Sato, A. (2005). Validation of the Japanese version of the primary and secondary psychopathy scale. The 69th Meeting of the Japanese Psychological Association, Tokyo, 10-12 September 2005.

Twenge, J. M., Baumeister, R. F., Tice, D. M., \& Stucke, T. S. (2001). If you can't join them, beat them: Effects of social exclusion on aggressive behavior. Journal of Personality and Social Psychology, 81, 1058-1069. doi:10.1037/0022-3514.81.6.1058

Ura, M., \& Takano, Y. (1995). An analysis of the relationship between the positive side and negative side of interpersonal relationship. The 36th Meeting of the Japanese Society of Social Psychology, Tokyo.

Warburton, W., Williams, K. D., \& Cairns, D. (2003). Effects of ostracism and loss of control on aggression. The 32nd Annual Meeting of the Society of Australasian Social Psychology, Sydney. 